\title{
La problématique de l'analyse des besoins de formation
}

L'analyse des besoins de formation et désormais considérée comme une figure imposée de la démarche de planification pédagogique. Dans ces colonnes ${ }^{1}$, une revue générale rappelait que, solon les modèles classiques de planification sys. tématique des activités de formation ${ }^{2}$, il sagis sait même de l'étape initiale du processus En formation professionnelle médicale, qu'elle soit initiale ou continue, on insiste aujourd'hui à juste titre pour que ces besoins de formation soient identifiés en faisant rééérence aux compé tences professionnelles visées 1,3 . Cedi implique une connaissance des problèmes de santé à résoudre par les futurs professionnels. Les modèles conventionnes de planification, d'ins piration souvent béhavioriste, décrivaient les termes du processus de manière linéaire, en faisant référence à des étapes successives $0 n$ tend à leur substituer aujourd'hui des approches beaucoup plusintégrées à pluseurs entrées LeBoterf parle à cet égard d'un « passage progressif d'une ingénierie séquentielle à une ingénierie « concourante » ou «s smultanée» de la forma$\operatorname{tion}^{4}{ }^{\Perp}$.

D ans cette livrai son du journal, J-M . Classe et coll. rapportent les résultats issus d'un travail préliminaire visant à identifier les besoins de formation pour les internes de gynécologie obs térique, en matière de cancérologie séno-gyné cologique. Dans une discussion fort loyale de leur étude, ils mettent en exergue un déficit concernant les outils méthodologiques employés. Le recours exclusif au questionnaire comporte d'évidentes limites, que les auteurs se proposent opportunément de pallier dans destravaux complémentaires Le répertoire des techniques utilisables dans une démarche d'analye des besoins se confond avec celui des méthodes employées en sciences humaines et sociales En faisant référence à une typologie développée dans le champ de l'évaluation de programme $e^{6}$, on peut ainsi recourir, successivement ou parallèlement, à différents procédés. Les techniques d'enquête (observation avec liste de vérification ou échelle d'appréciation, observation participante, ques tionnaire, entretien semi-structuré, analyse de contenu, ... ) permettent de recueillir des faits (par exemple des indicateurs de santé, des tâches et despratiquesprofessionnelles), desopinions ou des attitudes. Les techniques de communication (groupe de discussion, ronde D elphi, techniques sollicitant un groupe nominal ou exploitant un incident critique, ... ) permettent en outre de déterminer des priorités parmi les besoins $L a$ fiche pratique publiée dans ce numéro ${ }^{7}$ fournit un exemple de grille d'analyse utilisable pour évaluer les besoins en formation continue. Enfin, les techniques de planification (matrice décisionnelle, approche dite PERT (Program Evaluation and Review Technique), recours à un consultant ou à un comité de planification, ... ), permettent également de déterminer des priorités maisaussi d'exploiter opérationnellement les besoins recensés et d'en anticiper la scénarisation pédagogique.

En réalité, comme bien souvent en éducation, le problème n'et pasliéaux outilseux-mêmes mais à la pertinence de leur utilisation par rapport au but poursuivi et au contexte à prendre en compte. La problématiquen'est en effet pasauss univoque qu'elle ne paraît et ced est lié, notamment, à l'ambiguïté même de la notion de besoin. U ne fiche à usage didactique publiée récemment ne propose pasmoins de cinq définiti ons du concept d'analyse de besoins en formati on $^{8}$, qui se recoupent partiellement: moyen de déterminer les besoins individues de formation, identification de l'écart entre une performance actuelle et une performance souhaitée, identification des performances déficientes chez les personnes en formation, reconnaissance des diffé rences entre les compétences des étudiants et ce 
qui est requis pour conduire à bien une tâche professionnelle, moyen d'identifier cequelesétudiants, en tant que groupe, doivent apprendre. II reste toujours classiquede distinguer les besoins ressentis (ce que les personnes indiquent comme étant leurs besoins), les besoins exprimés (besoins identifiés lors de mises en situation), les besoins prescrits (définis par des experts) et les besoins démontrés (déduits d'une comparai son avec un groupe de référence) ${ }^{9}$. Cette typologie reprend d'ailleurs partiellement celle établie dès 1985 par Stufflebeam et coll., ${ }^{10}$ qui distingue quatre perspectives possibles pour l'évaluation des besoins en éducation. Cette réflexion reste aujourd'hui très pertinente et très féconde, notamment parce que ces auteurs analysent les avantages et les limites de chaque positionne ment. Ainsi, ils soulignent que la perspective normative, qui assimile de fait le besoin de formation à l'écart entre les performances observées et les performances souhaitées est généralement bien acceptée voire suggérée par les pouvoirs publics. Elle est facilement applicable aux situations pour lesquelles existent des normes, des standards et des critères; elletend en revancheà favoriser la prise en compte des problèmesqui se prêtent plusfacilement à la mesure, à privilégier la validité apparente à la validité sociale ou éducationnelle et favorise une approche réductrice et technocratique de l'analyse des besoins (checklists scores,... ). La perspective démocratique met l'accent sur le besoin de formation cons déré comme un changement dés ré par une majorité ou par un groupede référence. Ellepermet d'impliquer de nombreuses personnes dans leprocessus et de consi dérer avec équité plusieurs points de vue, ce qui peut être exploité pour induire une dynamique institutionnelle. En contrepartie, elle expose au risque démagogique de confusion entre besoin et préférence, dépend étroi tement du degré d'information des groupes et des personnes sollicités et peut conduire à orienter la détermination desbesoins danslesens d'une adaptation excessive aux contraintes, financières par exemple. La perspective diagnos tique conçoit le besoin de formation comme un objet dont l'absence ou l'inadéquation peut savérer néfasteet dont la mise en œuvre apporte des bénéfices. Elle permet l'identification des besoins de formation respectivement couverts ou non satiffaits et utilise une démarche logi que de recherche de la preuve, ce qui peut par contre conduire à privilégier les besoins non satisfaits caricaturaux et à négli ger d'améliorer les besoins déà couverts ; elle se révele en pratique haute ment subjective (il y a peu d'effets " démontrés » de modalités d'intervention en éducation) et postule souvent que certains besoins ont un caractère absolu. La perspective analytique, enfin, considère le besoin de formation comme une orientation grâce à laquelle on peut anticiper une amélioration, compte tenu de la situation actuelle. Elle repose sur un jugement informé et sapparente à une démarche systématique de résolution de problème ; elle met l'accent sur l'amélioration et non plus seulement sur la remédiation, implique une description compète et éclectique de la situation et ne dépend pas de standards a priori ; ses limites sont liées au fait qu'elle requiert des personnes ressourcestrès expé rimentées, faute desquelles ce modèle théorique peut se révéler difficile à opérationnaliser.

N orman ${ }^{3}$ insiste pour que l'on ne confonde pas besoins d'apprentissage et besoins éducationnels. Les uns sont identifiés par l'apprenant, idéale ment dans le contexte d'une démarche d'autoévaluation et d'une pratique réflexive; les autres sont déterminés, selon différentes approches, par les institutions ou par les structures professionnelles mandatées Le problème et dès lors double. D'un côté, l'autonomie des apprenants, c'es-à-dire leur capacitéà auto-évaluer et autodiriger leurs apprentissages, et contrairement à ce que postulait la perspective de la pédagogie dite d'adultes - encore dénommée courant andragogique -, n'est jamais auss performante qu'on le pense. Elle se révèle bien souvent déficiente et uneétude, publiéeic même, illustrait précisément la difficulté de médecins générali stes à identifier leurs besoins de formation dans le domaine de la gynécologi $e^{11}$. On sait d'ailleurs que ced est vrai autant en formation initiale qu'en formation continue ${ }^{3,12}$. De l'autre, les 
démarches d'analyse des besoins éducationnels, qui sont effectuées en sintéressant à des populations et à des groupes, et en raison notamment des limites évoquées précédemment, ne sont en mesure d'identifier que des besoinstrèsgénéraux, pas nécessairement suffisamment pertinents et opérationnels pour les individus ${ }^{12}$.

Ce constat souligne combien la problématique de l'évaluation des besoins ne se limite pas à la discussion méthodologique concernant les outils à employer et met en exergue la nécessité de mettre en œuvre une approche gl obale de la planification pédagogique. A distance d'une formation, l'identification préalable des besoins éducationnels et une action pédagogique mais aussi une démarche sociale et professionnelle $e^{3,13}$.

\section{Références}

1. Jean P. Pour une planification méthodique des activités de formation. Pédagogie M édicale 2001 ; 2 : 101-107.

2. Jean $P, D$ esM archais JE, D elorme $P$. Apprendre à enseigner les sciences de la santé Guide deformation pratique. M ontréal (QC) : Les Publications M édéva, 1993.

3. N orman GR, Shannon SI, Marrin M L. The need for needsassessment in continuing medical education. BMJ 2004 ; 328 : 999-1001.

4. Le Boterf G. Ingéniérie et évaluation des compé tences Paris: Editions d'organisation, 2002.

5. Classe JM, D escamps P, Sagot P, Levêque J, Body G, Philippe $\mathrm{H}-J$, Billaud $\mathrm{E}$. Contribution préliminaire à une procédure de recueil des besoins de formation pour les internes du D.E.S. de gynécologie-obsté trique en matière de cancérol ogi eséno-gynécologique. Pédagogie médicale $2004 ; 5$ : 147-158.

6. Nadeau MA. Les techniques particulières In : Nadeau M A. L'évaluation de programme Théorieet pratique. $Q$ uébec $(\mathrm{Q} C)$ : Les Presses de l'U niversité Laval, 1988 : 335-361.
En situation d'intervention pédagogique, quel qu'en soit le format, l'identification et la validation des besoins d'apprentissage individues résulte d'une confrontation des caractéristiques individuelles des apprenants et des compétences professionnelles visées; en tant que telle, cette interaction de l'apprenant avec ses professeurs et avec ses pairs et une pratique d'enseignement et d'apprentissage qui sollicite l'engagement profond despersonnes Au delà des outilset destechniques, le caractère consensuel et pédagogique ment correct du concept d'analyse des besoins de formation nedoit faire occulter ni sa finalité, ni ses exigences

Jean JOU QUAN mailto:jean.jouquan@chu-bret.fr
7. Agora-Formatiuon (dir. : R Picot) : Technique de détection et d'analyse des besoins de formation. $L a$ grilleFGP (fréquence, gravité, problèmes). Pédagogie M édicale $2004 ; 5$ : 185-186.

8. Hesketh EA, Laidlaw JM. Developing the teaching instinct. 4 : N edsassessment. M ed Teach 2002 ; 24 : 594-597.

9. Grant J. Learning needs assessment : assessing the need. BM J $2002 ; 324$ : 156-159.

10. Stufflebeam DL, M C Cormick CH, Brinkerhoff $\mathrm{RO}, \mathrm{N}$ elson CO. Conducting Educational $\mathrm{N}$ eeds Assessment. Boston (M A) : Kluwer-N ijhoff Publishing, 1985.

11. Levasseur G, Bagot C, H onnorat C. Q ue disent les médecins généralistes de leurs besoins de formation en gynécologie ? Pédagogie M édicale 2002 ; 3 : 1924.

11. N orman $G$. The adult learner : a mythical species. Acad M ed $1999 ; 74$ : 886-889.

13. H onnorat C, Levasseur G. Q uels besoins enseigner, quel besoin d'enseigner ? Pédagogie $M$ édicale $2001 ; 2$ : 26-30. 